\title{
Behavior of the sandwich beam subjected to bending as a function of the core density
}

\author{
Ružica R. Nikolić ${ }^{1,2,}$, Jelena M. Djoković ${ }^{3}$, Jan Bujnak $^{4}$, Katarina Živković ${ }^{1}$ \\ ${ }^{1}$ Faculty of Engineering, University of Kragujevac, Sestre Janjić 6, 34000 Kragujevac, Serbia \\ ${ }^{2}$ Research Center, University of Žilina, Univerzitna 8215/1, 01026 Žilina, Slovakia \\ ${ }^{3}$ Technical Faculty in Bor, University of Belgrade, V. Jugoslavije 12, 19210 Bor, Serbia \\ ${ }^{4}$ Faculty of Civil Engineering, University of Žilina, Univerzitna 8215/1, 01026 Žilina, Slovakia
}

\begin{abstract}
The sandwich beam can be considered as the multi-layered structure with a symmetrical cross-section. In this paper is assumed that the structure is created by periodical repetition of a unit cell. The influence of its size on the beam's static behavior in bending was analyzed. The variation of the unit cells number affects the size of the cell, so the static analysis was performed - the flexural stiffness and the beam's deflection were determined as functions of the unit cells number. The two configurations of the sandwich beams were considered: the beam with the constant cross-section along its length and the beam with the periodically variable cross-section. The graphs of the beam's flexural stiffness and deflection variations in terms of the unit cells number were obtained. It was concluded that after a certain number of the cells, the core's density does not further influence the behavior of the sandwich beam, under the given loading conditions. The conclusion from comparison of the two configurations is that the sandwich beam with the variable cross-section behaves somewhat better than the beam with the constant cross-section. The FEM analysis has verified all the conclusions from the analytical solution about the sandwich beams behavior when subjected to bending.
\end{abstract}

\section{Introduction}

Sandwich structures are the laminar structures that usually consist of the two thin, rigid plates - faces, which are separated by the softer layer - the core. The core material can be homogeneous isotropic foam, which contributes to the exceptionally small weight of the sandwich structure. Sometimes the core material is reinforced by the lateral fibers or the core consists of the thin-walled layers in the form of honeycomb or a truss.

Lightweight sandwich structures were studied by numerous investigators: Allen (1969) has set basics of the sandwich structures' design and analysis. Evans et al. (1998) have performed a survey of research on mechanical and thermal characteristics of the metal cellular systems. Brittain et al. (2001) have analyzed mechanical properties of the micro

"Corresponding author: ruzicarnikolic@yahoo.com 
plates with the truss core and proposed a new method for their manufacturing. Wicks and Hutchinson (2001) concluded that characteristics of the optimized sandwich plates with the truss core are superior to characteristics of plates with the honeycomb core or the stringerstiffened plates. Wicks and Hutchinson (2004) have further extended their analysis to sandwich plates with truss core subjected to fracture straining and combination of the bending moment and transversal force. Valdevit et al. (2004) were conducting the structural optimization of the sandwich plates with the truss core.

Sandwich structures have a wide application in different branches of industry, like the optical, biomechanical, microelectronics and highly resistant components, due to their high stiffness, superior strength, heat conductivity and small mass. To analyze the functionality and advantages of the sandwich structures, one should first comprehend the geometrical and mechanical factors, which influence the behavior of these structures.

The sandwich beam can be considered as the multi-layered structure with symmetrical cross-section. In this paper is assumed that the sandwich structure is created in such a way that a unit cell is periodically repeated. The attention is devoted to influence of the unit cell size, namely the number of the unit cells in the cross-section, on the static behavior of a beam in the bending loading conditions. The variation of the unit cells number affects the size of the cell, so the static analysis was performed - the flexural stiffness and the beam's deflection were determined as functions of the unit cells number, i.e. the core's density.

The two configurations of the sandwich beams were considered for the sake of comparison of their behavior under the same loading conditions: (i) the sandwich beam with the cross-section that is constant along the beam's length, which consists of the two faces while the core is made of the unit cell with the two square openings and (ii) the sandwich beams with the cross-section that changes periodically along the beam's length one cross section is full, while the other has square openings.

\section{Analytical solution of the problem}

In Figures 1 and 2 are shown the two considered configurations of the sandwich beam: in Figure 1 the sandwich beam with a cross section which is constant along the beam's length $L$ and in Figure 2 the sandwich beam with variable cross-section. The cross section of the former beam consists of the two flat plates, the beam's faces, upper and lower, of thickness $t_{f}$ and width $b$. The beam's core, of height $H_{C}$, is made of a unit cell with two square holes of size $a$. This is a sandwich structure with $n=1$, where $n$ represents a number of unit cells, i.e. the core's density. As can be seen in Figure 1 the structure with $n=2$ has eight square holes. If the structure could be built with sufficient (arbitrary) number of holes, $n$ could be practically limitless $(n=\infty)$. The cross section of the latter beam is periodically changing along its length. In the A-A section, the beam has the solid cross section, while in the B-B section the cross section has the square holes. The upper and the lower beam faces have the same thickness, $t_{f}$, while the beam's core height is $H_{C}$.

The faces and the core are made of different materials, so the elastic characteristics of the materials, the Young modulus and the Poisson's ratio, have indices 1 for the faces' material and 2 for the core's material, i.e. $E_{1}$ and $v_{1}$, and $E_{2}$ and $v_{2}$, respectively.

The differential equation of the beam's elastic (deflection) line reads:

$$
B^{(n)} \frac{d^{2} u(x)}{d x^{2}}=M(x),
$$

where $B^{(n)}$ is the bending stiffness, $u(x)$ is the deflection and $M(x)$ is the bending moment for the cross section defined by coordinate $x$. 


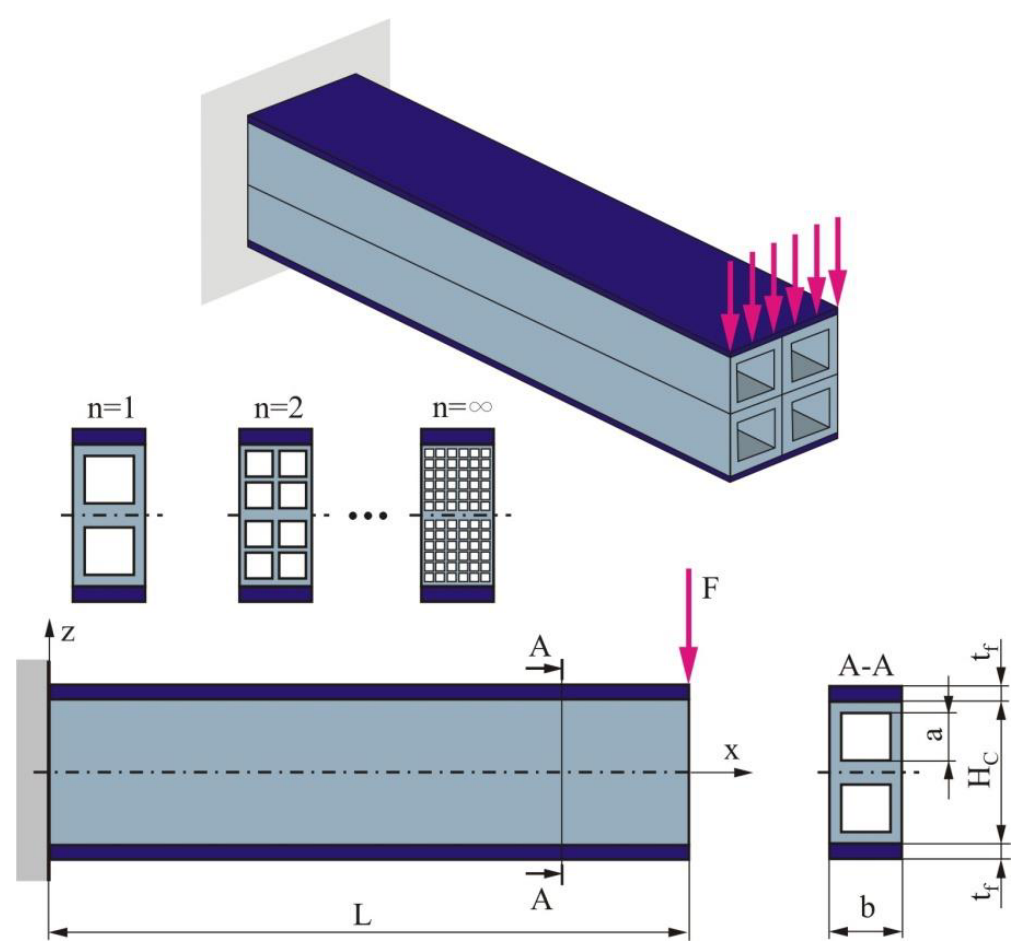

Fig. 1. Schematic presentation of the sandwich beam with the constant cross section.

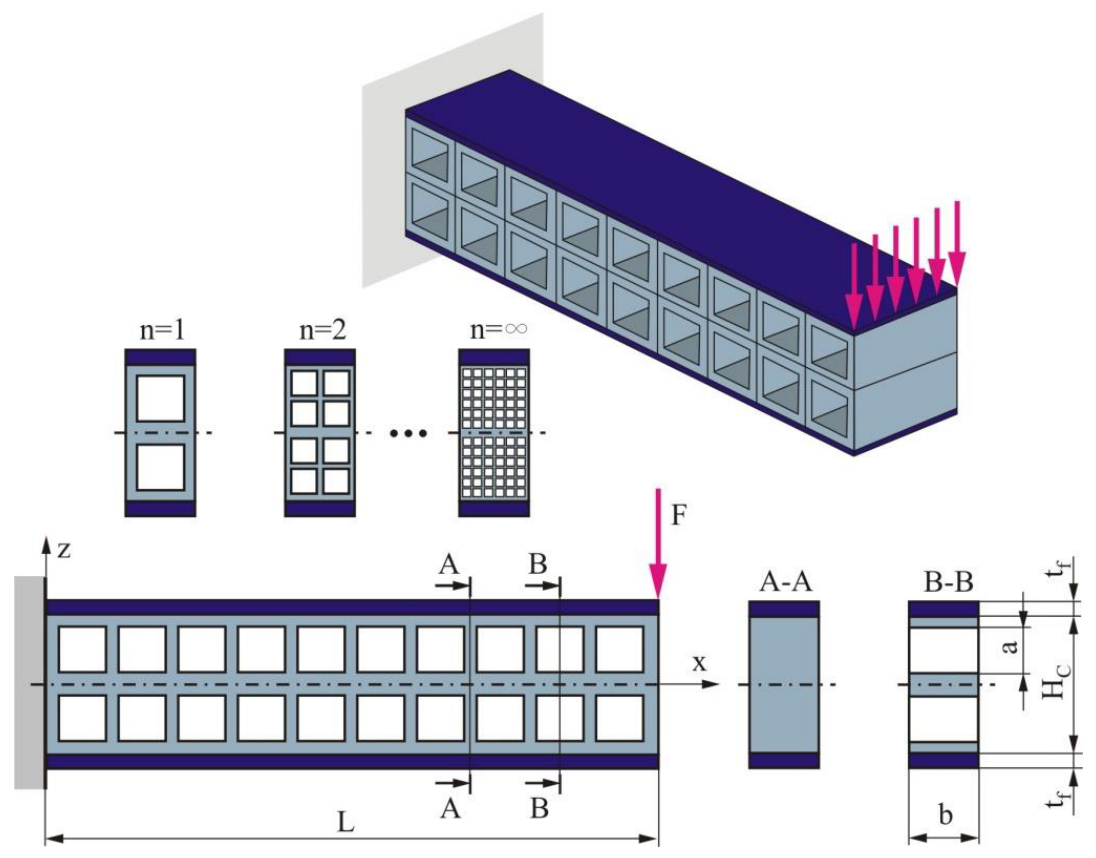

Fig. 2. Schematic presentation of the sandwich beam with the variable cross-section.

For the beam whose cross section is constant along its length, the bending stiffness is calculated as: 


$$
B_{c}^{(n)}=\sum E_{i} I_{i},
$$

while for the beam whose cross section is variable along its length the bending stiffness is calculated as:

$$
B_{v}^{(n)}=\left(\frac{1}{l} \int_{0}^{l} \frac{1}{B(x)} d x\right)^{-1}=l\left(\sum_{i} \frac{l_{i}}{B_{i}}\right)^{-1},
$$

where $l$ is the period's length.

For the loading conditions of beams presented in Figures 1 and 2, the largest deflection is obtained at the beams' free ends, i.e. for $x=L$, and can be calculated as:

$$
u_{\max }=\frac{F L^{3}}{3 B^{(n)}} .
$$

For the beam configuration presented in Figure 1 - the beam with the square cross section with the square holes, which does not vary along the beam's length, the bending stiffness in terms of the core's density is obtained as:

$$
\begin{aligned}
& B_{c}^{(n)}=\sum E_{i} I_{i}=2 E_{1} I_{1}+2 E_{2} I_{2}, \quad I_{1}=\frac{1}{3} b\left[\left(t_{f}+\frac{H_{c}}{2}\right)^{3}-\left(\frac{H_{c}}{2}\right)^{3}\right] \\
& I_{2}=\frac{1}{3} b\left(\frac{H_{c}}{2}\right)^{3}-\frac{1}{3} \frac{a}{n} \sum_{m=1}^{n} n\left[\left(\frac{(2 m-1) H_{c}}{4 n}+\frac{a}{2 n}\right)^{3}-\left(\frac{(2 m-1) H_{c}}{4 n}-\frac{a}{2 n}\right)^{3}\right] .
\end{aligned}
$$

For the beam configuration presented in Figure 2 - the beam with the square cross section with the square holes, which is varying along the beam's length, the bending stiffness in terms of the core's density is obtained as:

$$
\begin{aligned}
& B_{v}^{(n)}=2 E_{1} I_{1}+B_{\text {periodic }}^{(n)}, \quad B_{\text {periodic }}^{(n)}=2 E_{2} \frac{I_{2} I_{3} H_{c}}{\left(H_{c}-a\right) I_{3}+2 a I_{2}}, \\
& I_{1}=\frac{1}{3} b\left[\left(t_{f}+\frac{H_{c}}{2}\right)^{3}-\left(\frac{H_{c}}{2}\right)^{2}\right], \quad I_{2}=\frac{1}{3} b\left(\frac{H_{c}}{2}\right)^{3}, \\
& I_{3}=\frac{1}{3} b\left(\frac{H_{c}}{2}\right)^{3}-\frac{1}{3} \frac{a}{n} \sum_{m=1}^{n} n\left[\left(\frac{(2 m-1) H_{c}}{4 n}+\frac{a}{2 n}\right)^{3}-\left(\frac{(2 m-1) H_{c}}{4 n}-\frac{a}{2 n}\right)^{3}\right] .
\end{aligned}
$$

In Figure 3 is presented variation of the flexural stiffness in terms of the core density for both beam configurations and for the same loading conditions. Results were obtained according to equations (5) and (6) by application of the Mathematica ${ }^{\circledR}$ programming routine.

In Figure 4 is presented variation of the beam's free-end deflection in terms of the core density for both beam configurations and for the same loading conditions. Results were obtained according to equations (4), (5) and (6) by application of the Mathematica ${ }^{\circledR}$ programming routine. 


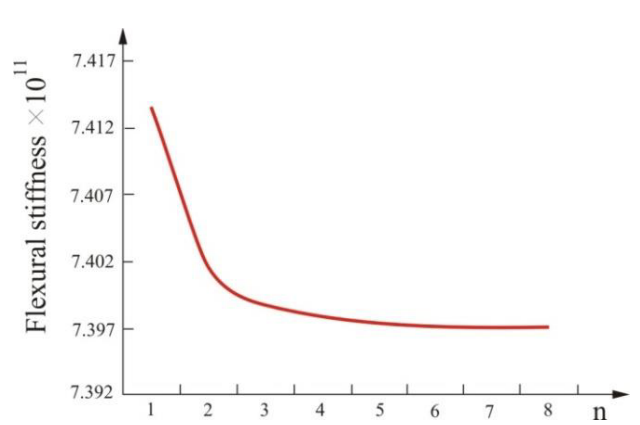

(a)

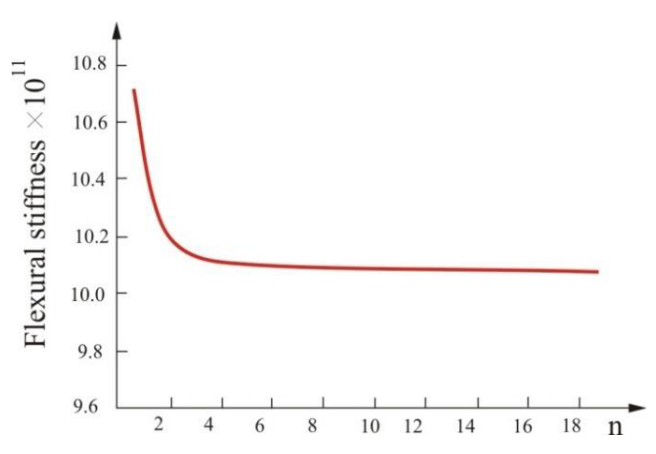

(b)

Fig. 3. Beam flexural stiffness in terms of the core density $n$ for the beam: (a) with the cross-section that is constant along the length; (b) with the cross-section that is variable along the length.

From Figure 3 one can notice that the flexural stiffness decreases with the core's density increase. It can also be noticed that after reaching the value of $n>5$, the stiffness remains almost constant. From Figure 4 one can notice that the value of the beam's free-end increases with increase of the beam's core density. Same as for the flexural stiffness, after reaching the value of $n>5$, the deflection also remains constant. From that one can conclude that further increase of the core density does not influence the behavior of the sandwich beam in the given loading conditions.

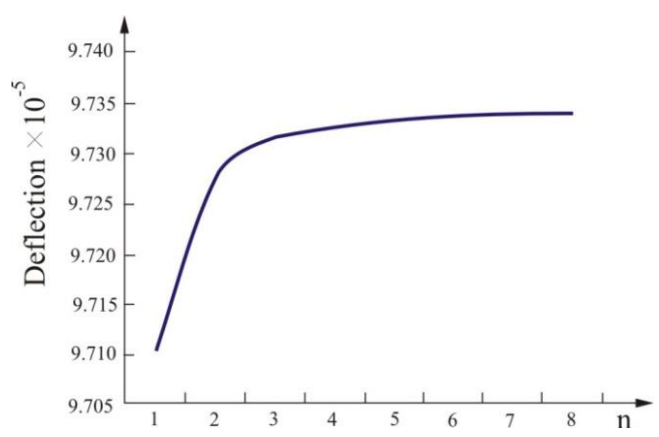

(a)

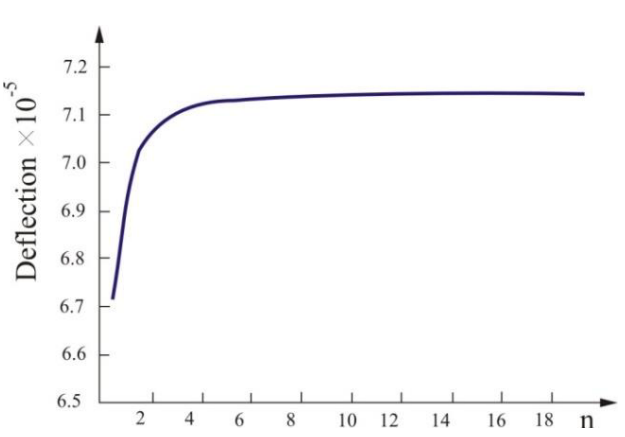

(b)

Fig. 4. The free-end deflection in terms of the core density $n$ for the beam: (a) with the cross-section that is constant along the length; (b) with the cross-section that is variable along the length.

\section{Finite Element Analysis of the problem}

For the purpose of verification of the analytical results, the FME analysis of the two beams' behavior was also preformed. In Figure 5 is presented the beam's free-end deflection, for both beam configurations, for three different values of the core's density. The ANSYS program was used for analysis within the package AutoDesk Invertor.

If one compares the flexural stiffness and the beam's free-end deflection for the two beam configurations, i.e. considers Figures 3, 4 and 5, one can conclude that the sandwich beam configuration with the variable cross-section is better than the configuration with the constant cross-section, for the same loading conditions. 

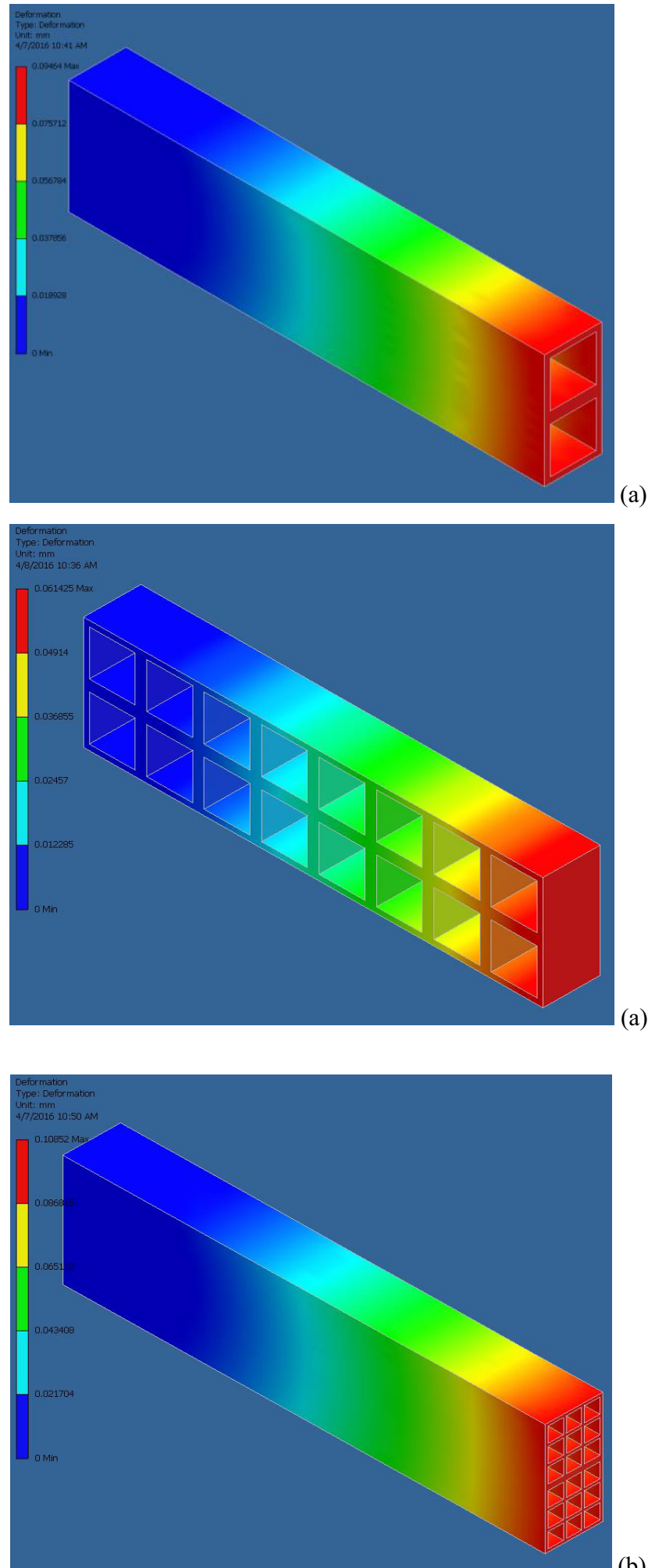

(b) 


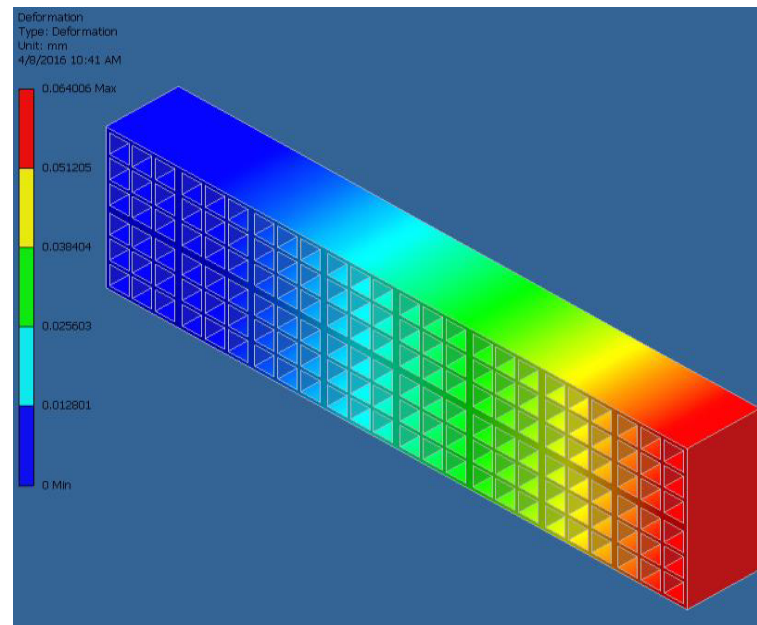

(b)
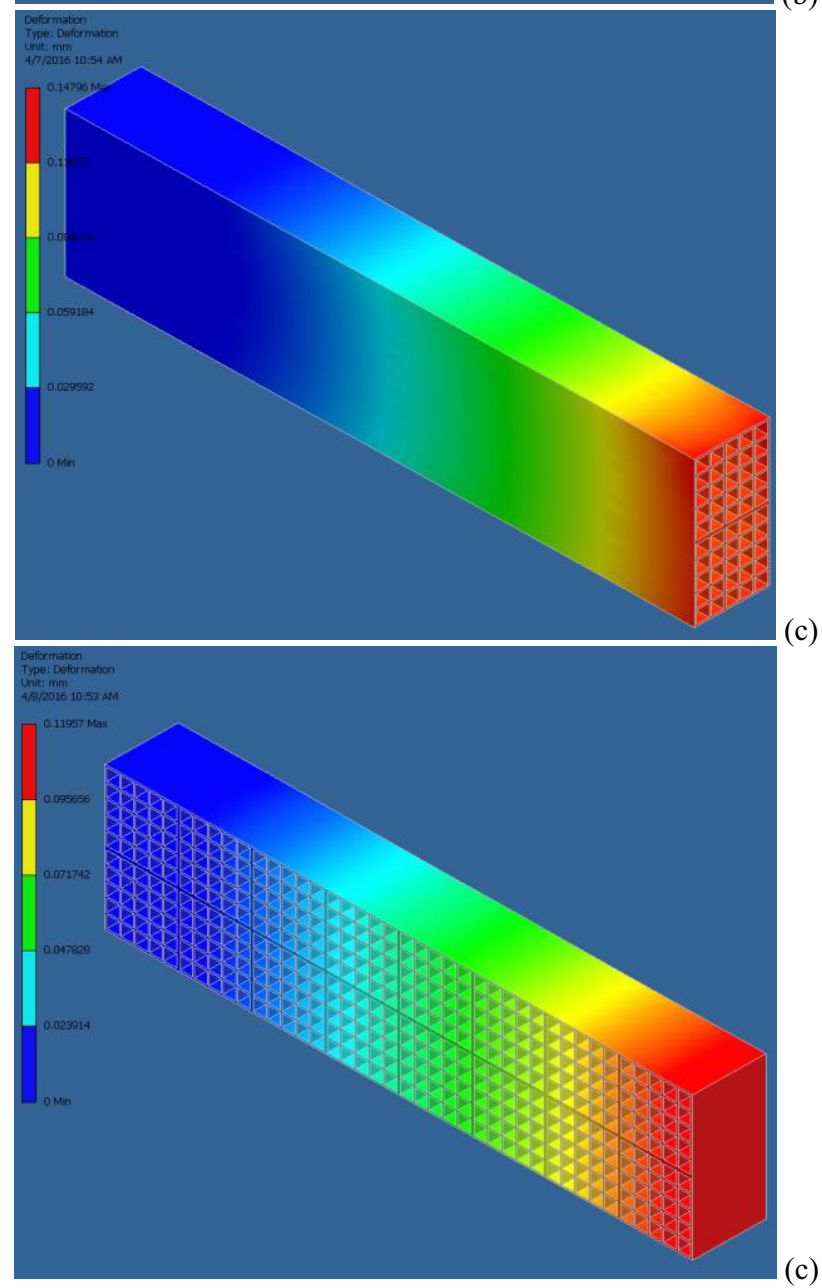

Fig. 5. The free-end deflection in terms of the core density $n$ for various core densities, for the beam with the constant cross-section (up) and for the beam with the variable cross-section (below):

(a) $n=1$, (b) $n=3$ and (c) $n=5$. 


\section{Conclusion}

In the paper is analyzed the influence of the core density on behavior of the two sandwich beam configurations subjected to bending. The two sandwich beams were considered as the laminar structures with the symmetrical cross-sections. The static analysis was performed to establish the influence of the core's unit cell size on the sandwich beams flexural stiffness and the beam's free-end deflection.

From results obtained both by analytical calculations and by the FME numerical analysis it was concluded that the core density does influence the sandwich beam behavior, but only until the number of the unit cells reaches the value of 5 . The further increase of the core's density (above $n=5$ ) seems to have no effect on behavior of the sandwich beams subjected to bending by the lateral force.

From comparison of the two beams' configurations behavior, it was also concluded that the sandwich beam with the variable cross-section exhibits better behavior than the sandwich beam with the constant cross-section, under the same loading conditions.

This research was partially supported by the Ministry of Education, Science and Technological Development of Republic of Serbia through Grants ON174001, ON174004 and TR32036 and by European regional development fund and Slovak state budget by the project "Research Center of the University of Žilina" - ITMS 26220220183. The authors are very grateful for this funding.

\section{References}

1. H.G. Allen, Analysis and Design of Structural Sandwich Panels (Pergamon Press, Oxford, 1969).

2. A.G. Evans, J.W. Hutchinson, M.F. Ashby, Prog. Mater. Sci 43(3), 171-221 (1998)

3. S.T. Brittain, Y. Sugimura, J.A. Schueller, A.G. Evans, G.M. Whitesides, J. Microelectromech. Syst. 10, 113-120 (2001)

4. N. Wicks and J.W. Hutchinson, Int. J. Sol. Struct. 38, 5165-5183 (2001)

5. N. Wicks and J.W. Hutchinson, Mech. Mater. 36, 739-751 (2004)

6. L. Valdevit, J.W. Hutchinson, A.G. Evans, Int. J. Sol. Struct. 41, 5105-5124 (2004) 